\title{
COMMENTARY
}

\section{Fusing Transcriptomics to Progressive Prostate Cancer}

\author{
Masaya Jimbo, ${ }^{*}$ Karen E. Knudsen, ${ }^{\dagger}$ and Jonathan R. Brody*
}

From the Division of Surgical Research, * Department of Surgery, The Jefferson Pancreas, Biliary, and Related Cancer Center, Sidney Kimmel Medical College, and the Departments of Cancer Biology, Urology, and Radiation Oncology, ${ }^{\dagger}$ Kimmel Cancer Center, Thomas Jefferson University, Philadelphia, Pennsylvania

CME Accreditation Statement: This activity ("ASIP 2014 AJP CME Program in Pathogenesis") has been planned and implemented in accordance with the Essential Areas and policies of the Accreditation Council for Continuing Medical Education (ACCME) through the joint sponsorship of the American Society for Clinical Pathology (ASCP) and the American Society for Investigative Pathology (ASIP). ASCP is accredited by the ACCME to provide continuing medical education for physicians.

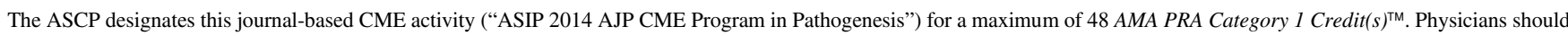
only claim credit commensurate with the extent of their participation in the activity.

CME Disclosures: The authors of this article and the planning committee members and staff have no relevant financial relationships with commercial interests to disclose.

Despite recent gains in extending survival of patients with metastatic disease, prostate cancer remains the most frequently diagnosed malignancy in the United States and is the second leading cause of cancer-related mortality among men. ${ }^{1}$ At the time of diagnosis, truly organ-confined disease can be readily treated through radical prostatectomy or radiation therapy, but there is no durable cure for disseminated disease, and metastatic prostate cancer remains uniformly fatal. Thus, the molecular linchpin as to how and why certain prostate cancers are indolent whereas others become more aggressive and metastatic is not well understood. ${ }^{1}$ Identifying a molecular signature to distinguish the behavior of individual cancers could save unwanted treatments and at the same time better select patients for effective therapies.

In this issue of The American Journal of Pathology, Yu et $\mathrm{al}^{2}$ devised a strategy to screen for these elusive molecular footprints (ie, fusion transcripts) expressed in prostate tumor cells using a variety of molecular techniques, including various sequencing and in situ hybridization assays. As a training set, the investigators performed whole-genome sequencing and total RNA sequencing on five prostate cancers and used four age-matched benign prostate samples (a total of 19 samples). Using the Fusioncatcher program, ${ }^{3}$ the group identified 76 fusion transcripts. Through the application of several filters and validation studies (ie, RTPCR, Sanger sequencing, and fluorescence in situ hybridization analyses), they identified eight novel cancer-specific transcripts (Figure 1). As a positive control, the investigators found the most established, previously published transcript TMPRSS2-ERG in two of the samples. ${ }^{4} \mathrm{Yu}$ et $\mathrm{al}^{2}$ could pave the way for a much needed biomarker panel and also a better understanding of the functional pathways involved in cancer cell invasion and metastasis.

\section{Historical Perspective}

High throughput genetic analysis on prostate cancer genomes has only been performed during the past five years. For example, in a landmark study by Berger et al, ${ }^{5}$ the investigators performed the first whole-genome sequencing analysis of human prostate cancer using seven primary patient tumors and paired normal tissues, and they found novel genomic rearrangements and deletions. Since then, a variety of nextgeneration sequencing approaches-including whole-genome sequencing, whole exome sequencing, and total RNA sequencing-have led to the discovery of numerous somatic mutations in the prostate cancer genome, including the presence of copy number alterations and recurrent fusion genes. ${ }^{6,7}$

The first fusion gene to be discovered in prostate cancer was identified by Tomlins et al. ${ }^{4}$ They used a bioinformatics approach to discover fusions of the prostate-specific, androgen-inducible TMPRSS2 with the oncogenic transcription factors of the ETS family (ETV1, ERG). This fusion gene, now known to be the most common genomic alteration in

Accepted for publication August 6, 2014.

Address correspondence to Jonathan R. Brody, Ph.D., Department of Surgery, Thomas Jefferson University, 1015 Walnut St., Ste. 611A, Philadelphia, PA 19107. E-mail: jonathan.brody@jefferson.edu

Disclosures: None declared. 


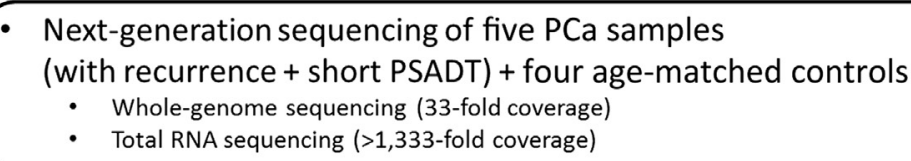

Eight fusion transcripts identified and validated

(TRMT11-GRIK2, SLC45A2-AMACR, MTOR-TP53BP1, LRRC59-FU60017,

TMEM135-CCDC67, KDM4-AC011523.2, MAN2A1-FER, CCNH-C5orf30)

Association of fusion transcripts with clinical outcomes in $90 \mathrm{PCa}+10$ controls

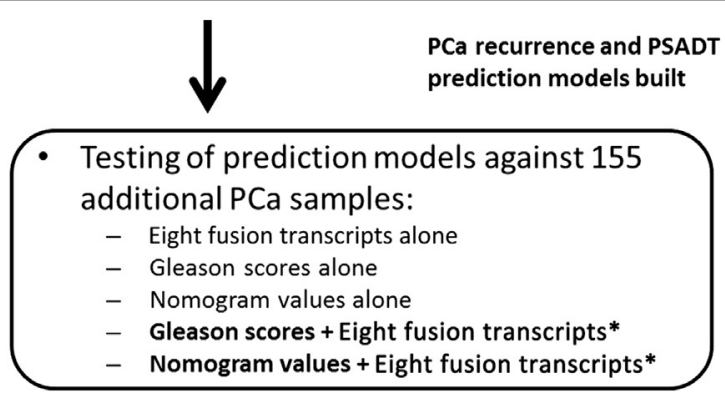

human prostate cancers $(>50 \%)$, allows for androgen-driven overexpression of oncogenic ETS. Since their discovery of the TMPRSS2-ETS fusion gene, many studies have been published regarding its clinical relevance (eg, its association with overall survival, recurrence, etc.). Unfortunately, many of the findings are contradictory with no overall consensus. ${ }^{8}$ In fact, Yu et $\mathrm{al}^{2}$ noted that TMPRSS2-ETS fusion status had no effect on their predictability model of prostate cancer recurrence. Although several other fusion genes have been discovered in prostate cancer since then, the current paper is the first to show strong evidence of the association between fusion transcripts and prognosis. ${ }^{2}$

\section{Molecular Insights}

The discovery of these fusion proteins raises a number of interesting mechanistic questions and potentially opens new lines of investigation. With the discovery of the novel fusion genes and their association with progressive prostate cancer, the natural question to ask is how the fusion events affect the biological functions of these genes, and therefore, directly contribute to tumorigenesis.

First, although it has been described previously, the presence of many fusion transcripts in normal or benign tissues is intriguing. ${ }^{9}$ Are these just random events that occur in normal healthy states? Are cells experimenting with these fusion products in an effort to become more tumorigenic? Or are these just innocuous, random events that occur at a high frequency with no impact on disease progression?

Second, some of the novel transcripts were detected with a relatively high frequency. For instance, TRMT11-GRIK2 and SLC45A2-AMACR were found in approximately 7\% of the samples. ${ }^{2}$ Interestingly, the TRMT11-GRIK2 fusion transcript predicts for a large truncation of TRMT11, a tRNA methyltransferase, and complete deletion of GRIK2, a tumor
Figure 1 Schematic of the work flow for the discovery of eight clinically relevant fusion products and their application in prediction models. Asterisks indicate significant improvement in the prediction rate of recurrence. $\mathrm{PCa}$, prostate cancer; PSADT, prostate specific antigen doubling time. suppressor and glutamate receptor. These data, along with expression analysis of the prostate samples, point toward this fusion gene generating a loss of function. In fact, the investigators point out that the only fusion gene resulting in detectable protein across all associated samples was MTORTP53BP1. The majority of the fusion genes resulted in products with low or undetectable protein expression.

Although elucidating the functions of the fusion genes is beyond the scope of the current article, the authors discuss the nature of two particular fusion genes: MAN2Al-FER and SLC45A2-AMACR. MAN2A1-FER is formed by the fusion of the Golgi apparatus-bound glycosyl transferase domain of $\alpha$ mannosidase 2 (MAN2A1) and the tyrosine kinase domain of feline sarcoma-related protein (Fer). $\alpha$-Mannosidase 2 is a key enzyme in the N-glycosylation pathway, abnormalities in which have been associated with disease progression and poor outcome in several cancer types. ${ }^{10}$ Fer is an oncogenic kinase whose expression is associated with more aggressive tumor characteristics in various tissues such as breast, lung, kidneys, and prostate. ${ }^{11-13}$ The authors speculate that the fusion product of these two genes may disrupt the proper glycosylation and phosphorylation of secreted or plasma membrane proteins, with far-reaching effects on all aspects of cancer cell biology.

SLC45A2-AMACR is formed by the fusion of solute carrier family 45 member 2 (membrane-associated transporter protein, or MATP), a protein involved in melanin biosynthesis, and $\alpha$-methylacyl-CoA racemase (P504S), an enzyme involved in branched-chain fatty acid metabolism. SLC45A2 has been shown to have protective roles in melanoma, and interestingly, fusions of the prostate-specific and androgen-regulated SLC45A3 with members of the ETS family have been previously discovered in prostate cancer specimens. ${ }^{7}$ Overexpression of $\alpha$-methylacyl-CoA racemase has been reported in several cancer types, and it is used as a diagnostic biomarker for prostate cancer. ${ }^{14}$ Although the authors could not explain the biological consequence of this 
fusion gene, they noted that tumor samples expressing this fusion gene showed the most highly aggressive clinicopathologic features.

Additional observations about these fusion transcripts are that either there is a large genomic distance between the genes, or these fusion transcripts are in the trans- orientation, which can theoretically only happen through a chromosome recombination event. In either case, the point is made that massive structural DNA rearrangements have to happen for these events to occur. This is consistent with previous findings, identifying gross chromosomal translocations and deletions as frequent in this disease type, as opposed to somatic point mutations. ${ }^{15}$ The fact that the majority of transcripts were either lowly expressed or undetectable also poses an interesting question about how a precursor or prostate cancer cell may efficiently silence a tumor suppressor gene. Alternately, if most of these fusion products are lowly expressed or undetectable, is there something specific about prostate cells that make them susceptible for chromosomal recombination events as a mode to silencing the expression of these genes compared to more conventional modes of gene silencing (such as point mutations or promoter methylation) found in other solid tumor types? Perhaps some regulatory mechanism becomes defective in these cells (eg, telomere shortening), which sets the stage for these recombination events and gene silencing.

Along with the line of questioning above, unanswered mechanistic questions include: Do these cells have shortened telomeres which allow for this genetic instability? ${ }^{16}$ Do prostate cancers that do not have the fusion phenotype, yet have similar clinical behavior, harbor inactivating, conventional mutations in the coding regions of these targeted-fusion genes or in other genes within the same signaling pathways? And finally and most importantly, what is the exact functional significance of these fusion events, and at what point in the prostate tumorigenesis (eg, high grade PIN) process are these events selected for?

\section{Clinical Impact and Future Studies}

The discovery that selected fusion transcripts may correlate with cancer progression is an advance, and it is consistent with the concept that genomic alterations are a hallmark of advanced disease. Determination of whether these transcripts serve as predictive or prognostic markers will be imperative to establish clinical utility. Notably, the presence of each individual product was quite low (approximately 3\% to 8\%) across prostate cancer specimens. ${ }^{2}$ Moreover, the cohort used assessed prostate specific antigen recurrence and doubling time after presumed definitive treatment (radical prostatectomy); the overall value of these endpoints for predicting aggressive behavior is unclear. Assessment of the fusion transcripts in a larger cohort and use of longitudinal analyses will be of benefit for integrating the present findings with other putative signatures of aggressive disease and determining comparative clinical utility. Such analyses will be of particular importance, because the study herein demonstrated a potential value for improving the predictive value of clinical models when the patient status of fusion genes is combined with current clinical metrics (ie, Gleason grade or Nomograms). Thus, although further investigation is needed to validate the capacity of the fusion transcripts to identify aggressive disease, this is an exciting advance directed toward an important clinical need.

\section{References}

1. Schrecengost R, Knudsen KE: Molecular pathogenesis and progression of prostate cancer. Semin Oncol 2013, 40:244-258

2. Yu YPDY, Chen Z, Liu S, Michalopoulos A, Chen R, Gulzar ZGYB, Cieply K, Luvison A, Ren B-G, Brooks JD JD, Nelson JP, Michalopoulos G, Tseng GC: J-H L: Novel fusion transcripts associate with progressive prostate cancer. Am J Pathol 2014, 184:2840-2849

3. Edgren H, Murumagi A, Kangaspeska S, Nicorici D, Hongisto V, Kleivi K, Rye IH, Nyberg S, Wolf M, Borresen-Dale AL, Kallioniemi O: Identification of fusion genes in breast cancer by paired-end RNA-sequencing. Genome Biol 2011, 12:R6

4. Tomlins SA, Rhodes DR, Perner S, Dhanasekaran SM, Mehra R, Sun XW, Varambally S, Cao X, Tchinda J, Kuefer R, Lee C, Montie JE, Shah RB, Pienta KJ, Rubin MA, Chinnaiyan AM: Recurrent fusion of TMPRSS2 and ETS transcription factor genes in prostate cancer. Science 2005, 310:644-648

5. Berger MF, Lawrence MS, Demichelis F, Drier Y, Cibulskis K, Sivachenko AY, et al: The genomic complexity of primary human prostate cancer. Nature 2011, 470:214-220

6. Hieronymus H, Schultz N, Gopalan A, Carver BS, Chang MT, Xiao Y, Heguy A, Huberman K, Bernstein M, Assel M, Murali R, Vickers A, Scardino PT, Sander C, Reuter V, Taylor BS, Sawyers CL: Copy number alteration burden predicts prostate cancer relapse. Proc Natl Acad Sci U S A 2014, 111:11139-11144

7. Nakagawa H: Prostate cancer genomics by high-throughput technologies: genome-wide association study and sequencing analysis. Endocr Relat Cancer 2013, 20:R171-R181

8. Gasi Tandefelt D, Boormans J, Hermans K, Trapman J: ETS fusion genes in prostate cancer. Endocr Relat Cancer 2014, 21:R143-R152

9. Li H, Wang J, Mor G, Sklar J: A neoplastic gene fusion mimics transsplicing of RNAs in normal human cells. Science 2008, 321:1357-1361

10. van den Elsen JM, Kuntz DA, Rose DR: Structure of Golgi alphamannosidase II: a target for inhibition of growth and metastasis of cancer cells. EMBO J 2001, 20:3008-3017

11. Ahn J, Truesdell P, Meens J, Kadish C, Yang X, Boag AH, Craig AW: Fer protein-tyrosine kinase promotes lung adenocarcinoma cell invasion and tumor metastasis. Mol Cancer Res 2013, 11:952-963

12. Ivanova IA, Vermeulen JF, Ercan C, Houthuijzen JM, Saig FA, Vlug EJ, van der Wall E, van Diest PJ, Vooijs M, Derksen PW: FER kinase promotes breast cancer metastasis by regulating alpha6- and beta1-integrin-dependent cell adhesion and anoikis resistance. Oncogene 2013, 32:5582-5592

13. Kawakami M, Morita S, Sunohara M, Amano Y, Ishikawa R, Watanabe K, Hamano E, Ohishi N, Nakajima J, Yatomi Y, Nagase T, Fukayama M, Takai D: FER overexpression is associated with poor postoperative prognosis and cancer-cell survival in non-small cell lung cancer. Int J Clin Exp Pathol 2013, 6:598-612

14. Ouyang B, Leung YK, Wang V, Chung E, Levin L, Bracken B, Cheng L, Ho SM: alpha-Methylacyl-CoA racemase spliced variants and their expression in normal and malignant prostate tissues. Urology 2011, 77:249.e1-249.e7

15. Baca SC, Prandi D, Lawrence MS, Mosquera JM, Romanel A, Drier Y, et al: Punctuated evolution of prostate cancer genomes. Cell 2013, 153:666-677

16. Meeker AK, Hicks JL, Platz EA, March GE, Bennett CJ, Delannoy MJ, De Marzo AM: Telomere shortening is an early somatic DNA alteration in human prostate tumorigenesis. Cancer Res 2002, 62:6405-6409 